\title{
Sistema con base en el Internet de las cosas para el control de riego en la agroindustria
}

\author{
Brenda Lilia Castro Coronado, Arnoldo Díaz-Ramírez, Verónica Quintero \\ Rosas, Mario Alberto Camarillo Ramos, Jorge Antonio Atempa Camacho \\ \{a12490431, adiaz, veronicaquintero, mario.camarillo, \\ jatempa\}@itmexicali.edu.mx
}

\begin{abstract}
Resumen. Las limitaciones de agua disponibles para los cultivos representa un tema de gran interés para la agroindustria. Maximizar la producción al mismo tiempo que se utiliza la cantidad óptima de agua es un problema que puede resolverse con el uso de las tecnologías de la información y comunicación. En este artículo se propone un sistema de riego de precisión que hace uso del paradigma del Internet de las Cosas. Los resultados de la evaluación del sistema propuesto permiten comprobar que puede contribuir en el uso eficiente del agua, al mismo tiempo que se mejora la producción.
\end{abstract}

Palabras clave: Internet de las cosas, agroindustria, control de riego.

\section{System based on the Internet of Things for Irrigation Control in Agribusiness}

\begin{abstract}
The limitations on the available water supply for the agroindustry is an issue of maximum concern. The goal of maximizing productivity using the optimum amount of water is a problem that can be addressed using the information and communications technologies. In this paper, a precision irrigation system, based on the Internet of Things paradigm, is introduced. The evaluation of the proposed system proves that it could be helpful in providing an efficient use of the available water, while the productivity is improved.
\end{abstract}

Keywords: Internet of things, agroindustry, irrigation control.

\section{Introducción}

La cantidad de agua existente en el suelo es fundamental para la producción eficiente de productos agrícolas. Monitorizar el nivel de humedad en el subsuelo de los cultivos es fundamental en la optimización de la producción, el uso racional 
del agua, la reducción de costos y el cuidado del medio ambiente. Por lo tanto, existe un gran interés en investigar el uso de la tecnología en el campo de la agroindustria, que ayude en la monitorización de los cultivos y el control de los procesos de producción. Una de las áreas que mas atención han recibido recientemente se denomina riego de precisión, que con base en el uso de la tecnología puede establecer el momento, la frecuencia y el tiempo de riego adecuados según las características del cultivo.

La falta de agua en los cultivos provoca un decremento en la calidad del producto, en el nivel de producción y eventualmente, la muerte de la planta. Por otra parte, el exceso de riego implica un desperdicio de agua, e incluso la muerte del cultivo. El riego de precisión permite determinar la cantidad y frecuencia óptima del riego.

En este artículo se propone un sistema de riego de precisión, que con base en el paradigma del Internet de las Cosas (IoT), pretende ser un apoyo en el control de riego en la agroindustria, optimizando el consumo del agua mediante la monitorización constante de la humedad del suelo.

El resto del documento está organizado de la siguiente manera: la Sección II discute la problemática del riego. En la Sección III se describe brevemente el paradigma del Internet de las cosas, mientras que la Sección IV describe el sistema propuesto. La Sección V está dedicada a discutir los resultados obtenidos de la evaluación del sistema propuesto. Finamente, en la Sección VI se presentan las conclusiones y el trabajo futuro.

\section{Problemática}

La agricultura es responsable del $70 \%$ de extracciones de agua dulce a nivel mundial [?], un recurso que en todas sus formas constituye solo el $3 \%$ del agua del mundo entero, mientras que el otro $97 \%$ es agua salada. Del $3 \%$ de agua dulce, aproximadamente del $2 \%$ está confinada en los casquetes glaciares de la Antártida y Groenlandia, a demasiados metros bajo tierra para ser accesible, o está contenida en la humedad del suelo, dejando tan solo el $1 \%$ de agua dulce del mundo disponible para uso humano. Además, el rápido desarrollo socioeconómico y urbanístico en los últimos años han hecho que el agua dulce sea insuficiente para satisfacer las necesidades básicas de la humanidad, en términos de usos agrícolas, industriales y urbanos. La escasez de agua se vuelve más aguda en regiones áridas y semiáridas debido a la distribución desigual de los recursos hídricos, tanto en el tiempo como en el espacio, y la competencia más fuerte para la demanda de agua entre diferentes sectores. Este problema puede reducirse utilizando el riego de precisión.

La agricultura de precisión requiere un conocimiento en tiempo real de los procesos en todas las áreas del campo. Las tecnologías utilizadas en el riego de precisión todavía están en etapa de desarrollo y constante mejora. El uso del riego de precisión implica el desarrollo de un sistema que gestione la máxima eficiencia en el uso del agua y maximizar la productividad [?]. 
Algunos de los parámetros básicos relacionados con el manejo eficiente del riego se describen a continuación [?], mismo que se muestran en la Fig. 1.

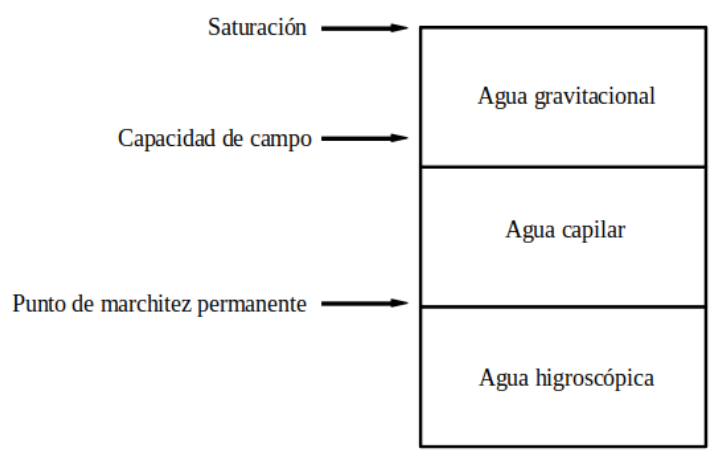

Fig. 1. Parámetros del agua en el suelo y tipos de agua en el suelo.

- Capacidad de campo. Es el contenido de agua o humedad máxima que puede retener el suelo. Esto por supuesto después de haber sido previamente drenado.

- Punto de marchitez permanente. Es el punto de humedad mínima que se alcanza en el que las plantas ya no pueden extraer más agua del suelo. Cuando la humedad está en este punto por mucho tiempo, puede provocarse que la planta ya no se recupere y eventualmente muera.

- Agua disponible para la planta. Es la cantidad de agua retenida en el suelo que esta disponible para que las plantas la extraigan.

- Contenido gravimétrico de agua. Es el contenido directo del agua en una muestra de suelo.

- Densidad aparente. Es la cantidad de masa de una muestra de suelo después de haber sido secada.

- Déficit permitido en el manejo del riego. Es el punto más bajo de contenido de agua en el suelo al cuál no se debe llegar para evitar el estrés en las plantas por falta de agua.

- Profundidad de la raíz. La profundidad a la que llegue la raíz de la planta es la que determina cuál es la cantidad de agua que va a requerir dicha planta.

Un sistema de riego de precisión es capaz de:

- Determinar el tiempo, la magnitud y el patrón espacial del riego para cumplir con el objetivo estacional (es decir, maximizar el rendimiento, la eficiencia del uso del agua o la rentabilidad).

- Aplicar exactamente (o lo más cerca posible) lo que se requiere de agua. 
- A través de experimentos de simulación, técnicas de aprendizaje autónomo o medición directa, conocer la magnitud y el patrón espacial de las aplicaciones de riego reales y las respuestas del suelo, de acuerdo a los tipos de cultivos.

- Utilizar estas respuestas para elaborar un plan de riego óptimo.

Adicionalmente, se han propuesto cuatro pasos esenciales en el proceso de control de riego [?], así como el uso algunas tecnologías para automatizar el proceso. Por ejemplo, en [?] se propone una arquitectura con base en IoT para la agroindustria, como puede observarse en la Fig. 2. Dicha arquitectura consta de cuatro componentes:

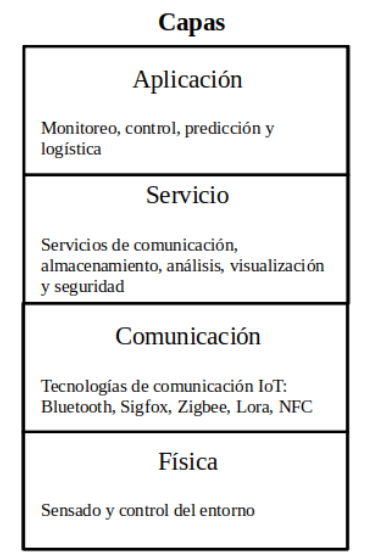

Fig. 2. Arquitectura propuesta con base en IoT [?].

1. Adquisición de datos. Un sistema de riego de precisión requiere un módulo de adquisición de datos del suelo y las condiciones del cultivo dentro de un campo.

2. Interpretación. Los datos recopilados se requieren interpretar, procesar y analizar.

3. Control. Debe contar con la capacidad de proveer de los recursos necesarios y ajustar el manejo del riego cuando se requiera.

4. Evaluación. Debe de haber una medición del rendimiento del sistema para obtener una retroalimentación que permita realizar mejoras para el próximo ciclo de riego.

La investigación en el riego de precisión inició en Estados Unidos a principios de 1990. Al principio el trabajo se centró en gran medida en la modificación de las máquinas de riego [?,?,?,?]. El interés de los investigadores europeos creció hasta la década de los 2000 y el énfasis se desplazó hacia el rendimiento de riegos. 
Los ejemplos de este trabajo incluyen Al-Karadsheh et al. [?], Camp et al. [?], Chavez et al. [?], King et al. [?] y Sadler et al. [?].

Trabajos recientes (Peters y Evett [?,?,?] y O'Shaughnessy et al. [?]) se han expandido para incluir el uso de termómetros infrarrojos montados en pivotes centrales para monitorizar las temperaturas del suelo, desarrollar protocolos para la programación y el control de riego automático en tiempo real. Otro trabajo reciente en la Universidad Estatal de Washington (Camp et al. [?]) se ha centrado en el desarrollo y prueba de sistemas de control digital que utilizan una computadora a bordo para implementar instrucciones transmitidas por radio para investigación de parcelas. También un trabajo adicional en Europa (Al-Karadsheh et al. [?]) examinó la respuesta de rendimiento a aplicaciones de agua no uniforme bajo sistemas de riego en movimiento. Adicionalmente, en Nueva Zelanda (Yule et al. [?], y Hedley y Yule [?,?]) se ha investigado el ahorro de agua y los beneficios económicos del riego de precisión utilizando pivotes centrales.

Algunos proyectos de investigación interesantes relacionados con el riego de precisión han sido desarrollados en Australia. Los grupos clave que participan en éste tema incluyen al Centro Nacional de Ingeniería en Agricultura (CNIA) de la Universidad de Southern Queensland, la Universidad de Melbourne y CSIRO. El CNIA también tiene un amplio programa de trabajo centrado en las tecnologías de detección para el riego de precisión [?].

\section{Internet de las cosas}

Cuando las computadoras salieron por primera vez al mercado, la tecnología no estaba creciendo tan rápido y no hubo muchas variaciones en los productos por algún periodo de tiempo. A medida que fueron cambiando las necesidades también fue avanzando la tecnología y se crearon soluciones para interconectar las computadoras. En un inicio, tenían que estar conectadas por cables y los usuarios estaban confinados a áreas de trabajo limitadas. Sin embargo, la tendencia se ha desplazado hacia la conectividad inalámbrica debido a la libertad que le da al usuario para moverse sin mucha restricción. Y los últimos años, la conectividad inalámbrica ha sido un área activa de investigación, y se han generado una gran cantidad de iniciativas de parte del gobierno y la industria. Esto ha motivado la creación de nuevos estándares y protocolos para alcanzar el objetivo de uniformidad y cohesión entre todas las tecnologías. Como resultado, hoy se cuenta con un conjunto de diversas de tecnologías de conectividad inalámbrica desde redes satelitales hasta sistemas celulares de área amplia [?,?].

El Internet de las Cosas es un paradigma que permite que los objetos cotidianos, de manera ubicua, sean capaces de monitorizar el entorno y transmitir a las personas u otros dispositivos, información de interés. En el IoT, las cosas u objetos pueden ser electrodomésticos, ropa, calzado, vehículos, entre otros. Estos objetos cuentan con sensores, sistemas inmersos, actuadores y radio-transmisores [?]. 
El paradigma de IoT busca interconectar objetos del mundo físico al mundo virtual, transformando la manera en la que se comunican entre sí, haciendo posible el intercambio de información sin necesidad de la intervención de un humano. Los protocolos de comunicación y estándares comúnmente usados dentro de éste paradigma incluyen: identificación por radio frecuencia (RFID) cerca de campo de comunicación (NFC ej. WLAN, ZigBee, Bluetooth) redes inalámbricas multisalto/redes de sensores, redes de área personal inalámbrica de bajo consumo bajo IPv6 (6loWPAN), máquina a máquina (M2M) y otras tecnologías tradicionales como IP, IPv6, etc [?].

En el caso particular de la problemática del riego de precisión, el paradigma de IoT se presenta como una valiosa alternativa para su uso en búsqueda de soluciones eficientes. Los avances en las tecnologías de sensores, sistemas inmersos, sistemas operativos, comunicación inalámbrica y protocolos para integración de IoT con la Computación en la Nube, permiten el diseño de arquitecturas IoT para la agroindustria. En la siguiente sección se presenta un sistema IoT con estas características.

\section{Sistema propuesto}

El contenido de humedad en las capas superficiales del suelo es un parámetro importante para muchas aplicaciones en las disciplinas de agricultura, hidrología y meteorología. Con la monitorización de la humedad del suelo se puede conocer la cantidad de agua que se encuentra en éste, lo cuál resulta fundamental para los agricultores. Con esta información se puede optimizar la producción definiendo una eficiente programación del riego. Por otra parte, puede determinarse la cantidad de agua a aplicar y cuándo aplicarla, evitando pérdidas de agua excesivas por percolación profunda o por escurrimientos, o bien evitar aplicar una cantidad insuficiente. Además, es posible reducir los impactos ambientales ya que el exceso de irrigación puede incrementar el consumo de energía y el desperdicio de agua, aumentar el movimiento de fertilizantes por debajo de la zona radicular, producir erosión, y provocar transporte de suelo y partículas de químicos a los canales de drenaje [?].

La irrigación es una práctica esencial en muchos sistemas de cultivos agrícolas en áreas semiáridas y áridas, y las aplicaciones y el manejo eficiente del agua son las principales preocupaciones. La determinación de cuándo irrigar requiere estimar el tiempo de riego para que no se produzcan reducciones de rendimiento debido al excesivo agotamiento de la humedad del suelo. Un método para la programación de riego consiste en medir o controlar el contenido de humedad del suelo. Para este propósito, los sensores de humedad del suelo se pueden usar para determinar el intervalo apropiado entre el riego, la profundidad de la humectación, la profundidad de extracción por las raíces y la idoneidad de la humectación [?,?]. Los sistemas de irrigación distribuidos en el campo y con base en el uso de sensores pueden ofrecer una solución potencial para respaldar la administración de irrigación específica. 


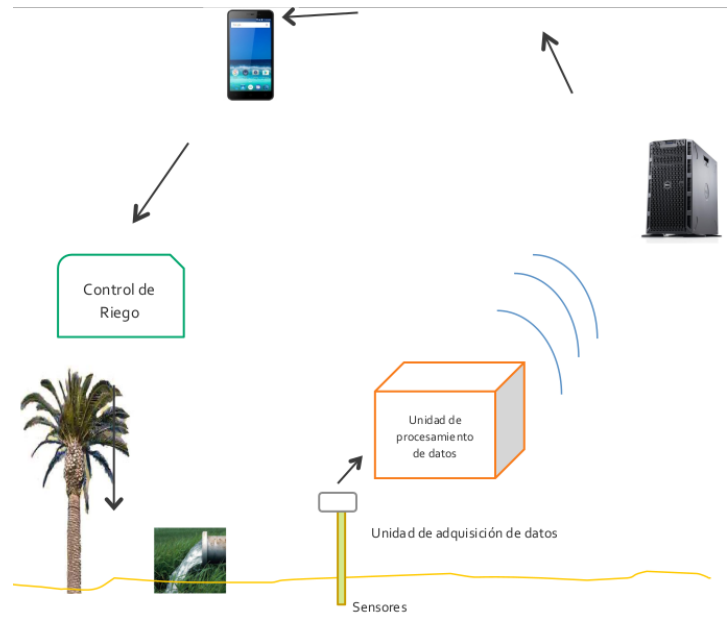

Fig. 3. Arquitectura del sistema propuesto.

En la Fig. 3 puede observarse la arquitectura del sistema propuesto. En primer lugar es necesario determinar los parámetros a monitorizar, relacionados con el nivel de humedad del suelo. Estos parámetros dependerán del tipo de suelo y cultivo. Por ejemplo, puede determinarse un umbral de humedad antes del punto de marchitez permanente, o medir también la capacidad de campo, como se muestra en la Fig. 1. Un vez determinados los parámetros es necesario seleccionar los sensores más adecuados para su monitorización, y en caso de no existir, diseñar una interfaz de hardware que pueda obtener los parámetros previamente determinados. Al módulo que recaba la información de los sensores se le denomina Unidad de Adquisición de Datos. La información recabada por los la Unidad de Adquisición de Datos debe ser pre-procesada o normalizada. Estas operaciones se llevan a cabo en la Unidad de Procesamiento de Datos, que es un sistema embebido con capacidades de comunicación inalámbrica. Los datos pre-procesados son enviados a un dispositivo con mayor capacidad de cómputo, en el que se ejecuta un proceso denominado Servidor, que recibirá las mediciones obtenidas por un conjunto de sensores a través de las Unidades de Procesamiento de Datos. El proceso servidor, que recibe las mediciones de los sensores, las almacena localmente y en la Nube, procesa los datos y lleva a cabo un análisis de los mismos. Con esta información decide las acciones a seguir relacionadas con el control del riego. Por ejemplo, notificar al usuario cuando un sector del cultivo tiene exceso o deficiencia de agua, o recomendar un programa de riego. Adicionalmente, se propone el desarrollo de una aplicación móvil que permita al usuario consultar, en tiempo real, los niveles de humedad de sus cultivos, o recibir alertas de situaciones anómalas. Finalmente, se propone que sea un sistema de ciclo cerrado, ya que se considera que el usuario sea capaz de activar el sistema de riego desde la aplicación móvil, con base a las recomendaciones del sistema. De igual manera, que pueda definir o ajustar el programa de riego con 
Brenda Lilia Castro Coronado, Arnoldo Díaz-Ramírez, Verónica Quintero Rosas, et al.

el apoyo de técnicas de aprendizaje autónomo, utilizando los datos obtenidos por el sistema.

\section{Prototipo}

Para evaluar la arquitectura del sistema propuesto, se desarrolló el prototipo propuesto. Sus componentes se explican a continuación:

\subsection{Unidad de Adquisición de Datos}

Unidad de Adquisición de Datos s epresetna en la Figura 4. Para monitorear los niveles de humedad en el suelo se seleccionó un sensor modelo Watermark [?]. Este sensor, patentado por la compañía Irrometer, Inc., es un dispositivo de resistencia eléctrica de estado sólido que se utiliza para medir la tensión del agua del suelo. A medida que la tensión cambia con el contenido de agua, la resistencia también cambia, correlacionando dicha resistencia a centibares (kilopascales) de la tensión del agua del suelo. Está diseñado para ser un sensor permanente, colocado en el suelo a monitorear y «leído» las veces que sea necesario con un dispositivo portátil o estacionario.

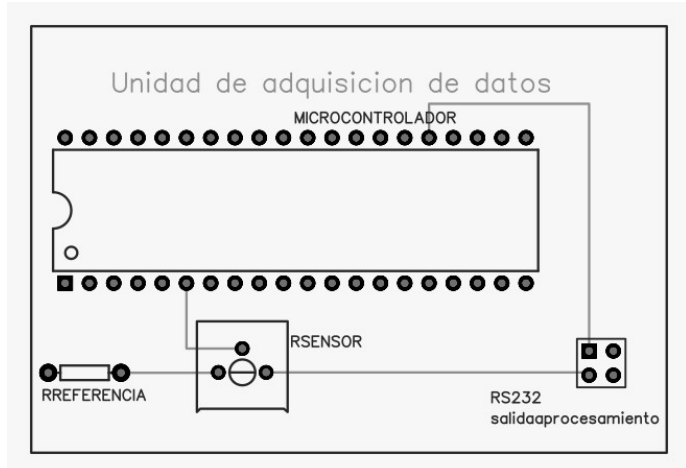

Fig. 4. Unidad de adquisición de datos.

Este sensor permite la conversión directa de la resistencia del sensor en ohms y la temperatura en valores potenciales para definir el agua del suelo. El fabricante proporcionó una tabla con los valores de resistencia y centibares. A partir de la cuál se utilizó un ajuste de curvas para acceder a las siguientes ecuaciones:

Para $R \leq 1 \mathrm{Kohm}$

$P=-20 *[R *(1+0,018 *(T-24))-0,55]$.

Para 1 Kohm $<R \leq 8$ Kohm 
$P=(-3,213 * R-4,093) /(1-0,009733 * R-8,01205 * T)$.

Para $R>8$ Komh

$P=-2,246-5,239 * R *(1+0,018 *(T-24))-0,06756 * R^{2} *(1+0,018 *(T-24))^{2}$.

Aquí $\mathrm{P}$ es el potencial hídrico del suelo, $\mathrm{R}$ es la resistencia del sensor, $\mathrm{T}$ es la temperatura del suelo.

Éste sensor se encuentra conectado a un microcontrolador serie PIC16F1779 [?] que cuenta con amplificadores operacionales y un convertidor analógicodigital para acondicionar la señal. Éste microcontrolador es el que se encarga de tomar las mediciones del sensor y mandarlas a la unidad de procesamiento de datos vía RS-232.

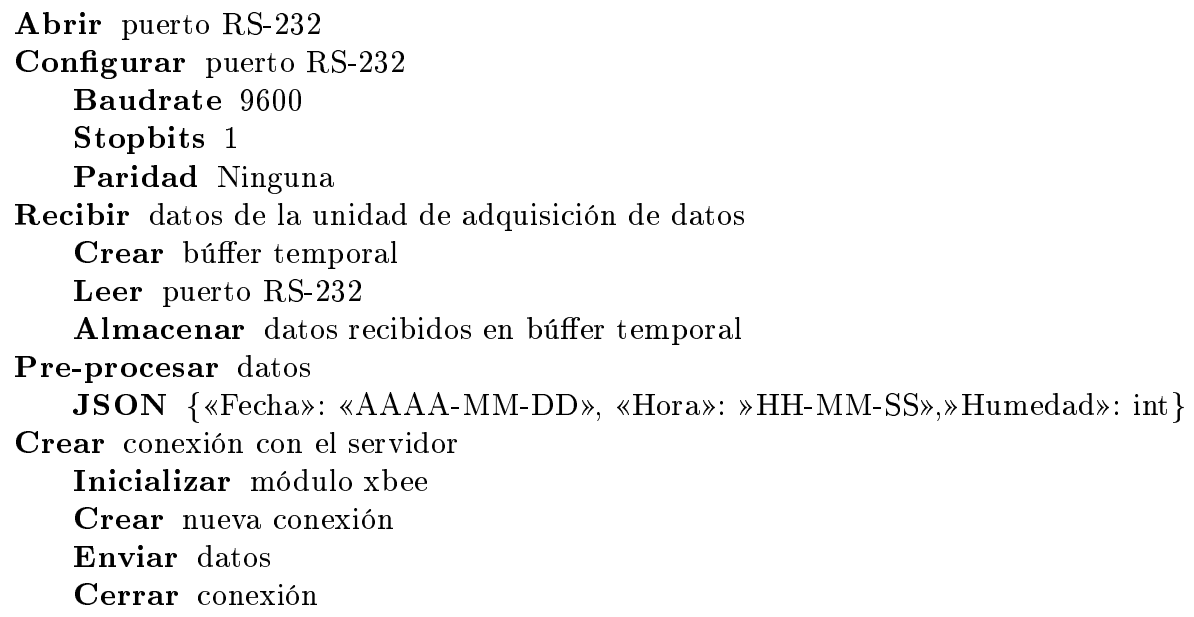

Algorithm 1: Unidad de procesamiento de datos.

\subsection{Unidad de Procesamiento de Datos}

Con la información recibida por el módulo de adquisición de datos, éste módulo realiza una normalización y un pre-procesamiento de los datos. Para el envío de la información se utilizó JSON, que es un formato ligero para el intercambio de datos. Para facilitar su uso, se utilizó la biblioteca de json-c [?]. Después del pre-procesamiento de los datos se envían al proceso servidor utilizando los protocolos de comunicación para WPAN denominados ZigBee e IEEE 802.15.4.

En este caso, se hizo uso de la biblioteca libxbee [?], la cuál es ligera y especialmente creada para la intercomunicación con dispositivos xbee. Ésta biblioteca hace interfaz con un módulo xbee modelo S2C de la compañía Digi International, Inc. [?]. La biblioteca implementa el protocolo Zigbee [?], especialmente diseñado 
Brenda Lilia Castro Coronado, Arnoldo Díaz-Ramírez, Verónica Quintero Rosas, et al.

Crear conexión con la unidad de procesamiento de datos

Inicializar módulo xbee

Crear conexión

Crear búffer temporal

Almacenar datos recibidos en búffer temporal

Almacenar datos recibidos en la base de datos

Crear conexión

Seleccionar base de datos

Insertar datos

Cerrar conexión

Post-procesar datos de acuerdo a las normativas de Carriots

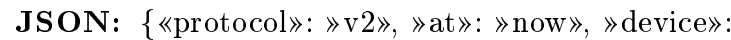
$»$ midispositivo@usuario.usuario»,《data»: \{«Fecha»: «AAAA-MM-DD», «Hora»: »HH-MM-SS»,»Humedad»: int $\}$ \}

Enviar datos al servidor MQTT de Carriots

Crear nueva instancia MQTT

Crear conexión

Enviar datos

Cerrar conexión

Algorithm 2: Servidor

para redes de control y sensores que utiliza en las capas inferiores al estándar IEEE 802.15.4 [?]. Una de las ventajas de ZigBee es que los dispositivos pueden comunicarse entre sí o actuar como repetidores si el servidor esta fuera del alcance del transmisor del nodo. En el Algoritmo 1 se muestra el pseudo-código utilizado en la Unidad de Procesamiento de Datos.

\subsection{Servidor}

Es un proceso desarrollado en lenguaje $\mathrm{C}$ que se ejecuta en una computadora con mayores recursos. En el prototipo se utilizó el sistema operativo Linux (Ubuntu v18.04 [?]), el cuál recibe la información pre-procesada y concluye su procesamiento usando igualmente la biblioteca json-c. L información recibida se almacena en una base de datos para que esté disponible para consulta utilizando el sistema administrador de bases de datos MariaDB [?], debido a que es rápido, escalable y robusto, compatible con MySQL, con un amplio ecosistema de motores de almacenamiento. Además de ser código abierto, tiene el soporte de otras herramientas que lo hacen muy versátil. También, con los datos ya debidamente normalizados, procesados y estructurados, se continua con el proceso de almacenamiento en la nube utilizando los servicios de un proveedor de computación en la nube.

En este caso, se utilizó Carriots [?]. El envío de información al proveedor de Computación en la Nube se lleva a cabo por medio del uso de MQTT [?], que es un protocolo de comunicación de tipo publisher-subscriber, extremadamente ligero y práctico, diseñado para dispositivos de pocos recursos o redes de bajo 
ancho de banda. MQTT es un protocolo diseñado para ser utilizado en aplicaciones IoT que se integran con la Computación en la Nube. En el Algoritmo 2 puede observarse el pseudo-código del proceso servidor.

\subsection{Nube}

La nube se presenta en la Figura 5. Carriots es una plataforma inteligente de arquitectura abierta, orientada a aplicaciones IoT. Carriots permite recopilar y almacenar datos de dispositivos conectados, crear aplicaciones potentes, implementar y escalar prototipos a miles de dispositivos. Además, permite el análisis de los datos y envío de notificaciones vía correo electrónico o mensaje de texto (ver Fig. 6).. Ésta plataforma si cuenta un costo mensual dependiendo de los dispositivos y los requerimientos del usuario. Para fines de pruebas del prototipo se utilizó la versión gratuita la cuál permite acceder a la plataforma y gran parte de sus herramientas hasta cierto límite.

\subsection{Notificaciones}

Una de las facilidades que ofrece la plataforma de Carriots, es la de enviar mensajes de texto o correo electrónico para realizar notificaciones con base a los parámetros establecidos. Éstos por supuesto por ser la versión gratuita tienen un límite el cuál fue suficiente para poder realizar las pruebas con el prototipo diseñado.

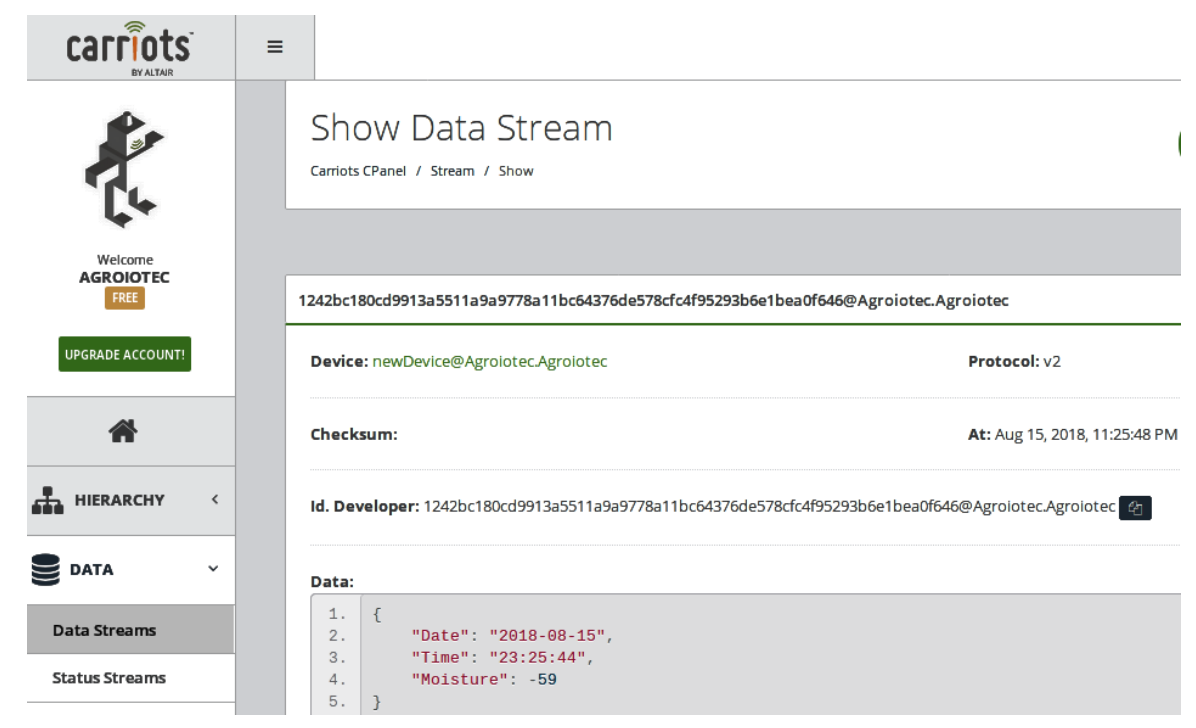

Fig. 5. Nube de Carriots. 
Brenda Lilia Castro Coronado, Arnoldo Díaz-Ramírez, Verónica Quintero Rosas, et al.

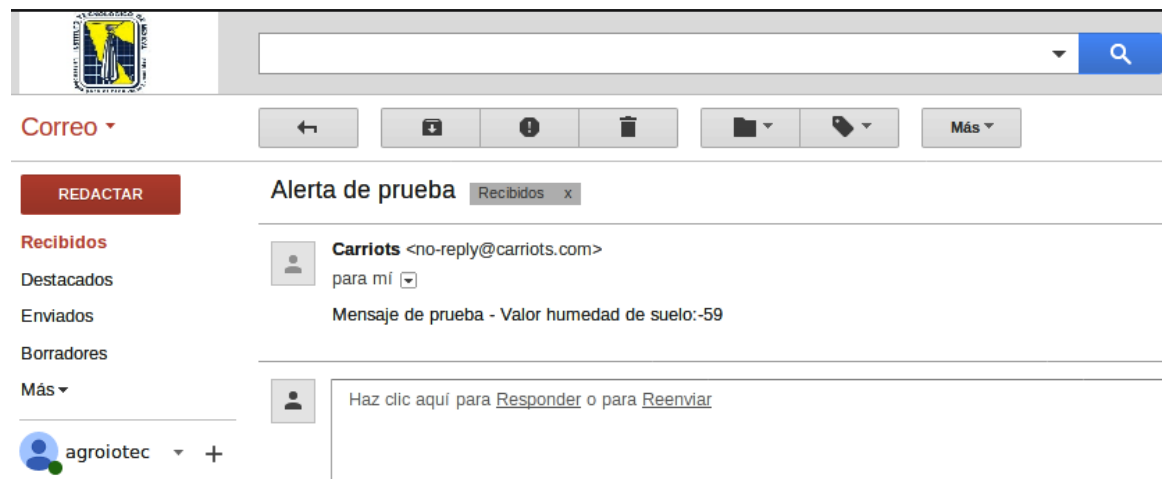

Fig. 6. Notificación de correo electrónico por Carriots.

\subsection{Resultados}

Al evaluar el desempeño prototipo se obtuvieron resultados satisfactorios, pudiendo realizar el sensado del nivel de humedad del suelo, hasta el envío de notificaciones de prueba vía correo electrónico sobre los estados de la humedad del suelo.

\section{Conclusiones y trabajo futuro}

En este artículo se presentó un sistema para el control de riego en la agroindustria, con base en el paradigma del Internet de la Cosas. El sistema propuesto fue capaz de monitorizar en tiempo real los niveles de humedad en los cultivos y de emitir alertas en caso de detectar condiciones anómalas. La evaluación de la propuesta sugiere que el uso de IoT en la agroindustria permite hacer un uso racional del agua.

Como trabajo futuro previsto se planea investigar y analizar otros proveedores de servicios computación en la nube para conocer ventajas y desventajas de cada uno de ellos. Adicionalmente, evaluar sistemas operativos para sistemas embebidos e implementar el mas conveniente en el sistema propuesto.

También se tiene previsto integrar técnicas de aprendizaje automático y utilizar la información recabada para hacer una retroalimentación y poder planear mejor los próximos riego de manera inteligente.

\section{Referencias}

1. Al-Karadsheh, E., Sourell, H., Krause, R.: Precision irrigation: New strategy irrigation water management. In: Proceedingd of the Conference on International Agricultural Research for Development, Deutscher Tropentag, Wiltzenhausen, Germany. pp. 9-11 (2002) 
2. Ashraf, M., Safdar, M.E., Shahzad, S.M., Aziz, A., Piracaha, M.A., Suleman, M., Ahmad, M.B.: Challenges and opportunities for using wastewater in agriculture: a review. Journal of Applied Agriculture and Biotechnology 2(2), 1-20 (2018)

3. Atzori, L., Iera, A., Morabito, G.: The internet of things: A survey. Computer Networks 54(15), 2787-2805 (Oct 2010)

4. Bronson, K., Booker, J., Bordovsky, J., Keeling, J., Wheeler, T., Boman, R., Parajulee, M., Segarra, E., Nichols, R.: Site-specific irrigation and nitrogen management for cotton production in the southern high plains. Agronomy Journal 98(1), 212-219 (2006)

5. json c: github.com/json-c/json-c, https://github.com/json-c/json-c

6. Camp, C.R., Sadler, E.J., Evans, R.G.: Precision water management: current realities, possibilities, and trends. Handbook of Precision Agriculture, Haworth Press, Inc., Bunghamton, NY pp. 153-184 (2006)

7. Carriots: www.carriots.com, https://www.carriots.com/

8. Chavez, J.L., Pierce, F.J., Matthews, G.R., Elliott, T.V., Evans, R.G., Kim, Y.: Performance of a continuous move irrigation control and monitoring system. In: 2006 ASAE Annual Meeting. p. 1. American Society of Agricultural and Biological Engineers (2006)

9. Da Xu, L., He, W., Li, S.: Internet of things in industries: A survey. IEEE Transactions on industrial informatics 10(4), 2233-2243 (2014)

10. Digi: www.digi.com, https://www.digi.com/

11. Enciso, J.M., Porter, D., Peries, X.: Uso de sensores de humedad del suelo para eficientizar el riego. University of Texas System, Austin, TX (2007)

12. Evans, R.G., Han, S., Kroeger, M., Schneider, S.M.: Precision center pivot irrigation for efficient use of water and nitrogen. Precision agriculture (precisionagricu3), 75-84 (1996)

13. Ghodake, R., Jogade, S., Misal, D.: Bluetooth technology: An overview (2018)

14. Haverkort, A., Vos, J., Booij, R.: Precision management of nitrogen and water in potato production through monitoring and modelling. In: XXVI International Horticultural Congress: Potatoes, Healthy Food for Humanity: International Developments in Breeding, 619. pp. 213-224 (2002)

15. Hedley, C.B., Yule, I.J.: Soil water status mapping and two variable-rate irrigation scenarios. Precision Agriculture 10(4), 342-355 (2009)

16. Hedley, C., Yule, I.: A method for spatial prediction of daily soil water status for precise irrigation scheduling. Agricultural Water Management 96(12), 1737-1745 (2009)

17. IEEE: www.iee.org, https://www.ieee.org/

18. Irrometer: www.irrometer.com, http://www.irrometer.com/

19. Kelly, R., Strong, W., Jensen, T., Butler, D.: Application of probability analysis to assess nitrogen supply to grain crops in northern australia. Precision Agriculture 5(2), 95-110 (2004)

20. Kim, Y., Evans, R.: Software design for wireless sensor-based site-specific irrigation. Computers and Electronics in Agriculture 66(2), 159-165 (2009)

21. Kim, Y., Evans, R.G., Iversen, W.M.: Remote sensing and control of an irrigation system using a distributed wireless sensor network. IEEE transactions on instrumentation and measurement 57(7), 1379-1387 (2008)

22. King, B., Wall, R., Kincaid, D., Westermann, D.: Field testing of a variable rate sprinkler and control system for site-specific water and nutrient application. Applied engineering in agriculture 21(5), 847-853 (2005)

23. libxbee: github.com/attie/libxbee3, https://github.com/attie/libxbee3 
24. MariaDB: mariadb.org, https://mariadb.org/

25. Mehta, A.: Bluetooth technology: an exploratory study of the analysis and implementation frameworks. Riset Journal of Science \& Engineering 1(1) (2018)

26. Microchip: www.microchip.com, https://www.microchip.com/

27. MQTT: mqtt.org, mqtt.org

28. O'Shaughnessy, S.A., Evett, S.R., Colaizzi, P.D., Howell, T.A.: Soil water measurement and thermal indices for center pivot irrigation scheduling. In: Proceedings of the 2008 Irrigation Show and Conference. pp. 2-4 (2008)

29. Peters, R.T., Evett, S.R.: Complete center pivot automation using the temperature-time threshold method of irrigation scheduling. In: 2004 ASAE Annual Meeting. p. 1. American Society of Agricultural and Biological Engineers (2004)

30. Peters, R.T., Evett, S.R.: Automation of a center pivot using the temperaturetime-threshold method of irrigation scheduling. Journal of irrigation and drainage engineering 134(3), 286-291 (2008)

31. Peters, R., Evett, S.: Spatial and temporal analysis of crop conditions using multiple canopy temperature maps created with center-pivot-mounted infrared thermometers. Transactions of the ASABE 50(3), 919-927 (2007)

32. Sadler, E., Evans, R., Stone, K., Camp, C.: Opportunities for conservation with precision irrigation. Journal of soil and water conservation 60(6), 371-378 (2005)

33. Smith, R., Baillie, J., McCarthy, A., Raine, S., Baillie, C.: Review of precision irrigation technologies and their application (2010)

34. Talavera, J.M., Tobon, L.E., Gomez, J.A., Culman, M.A., Aranda, J.M., Parra, D.T., Quiroz, L.A., Hoyos, A., Garreta, L.E.: Review of iot applications in agroindustrial and environmental fields. Computers and Electronics in Agriculture 142, 283-297 (2017)

35. Ubuntu: www.ubuntu.com, https://www.ubuntu.com/

36. Yule, I., Hedley, C., Bradbury, S.: Variable-rate irrigation. In: 12th Annual Symposium on Precision Agriculture Research \& Application in Australasia. Sydney (2008)

37. Zigbee: www.zigbee.org, http://www.zigbee.org/ 